\title{
Win X-ray, The Monte Carlo Program for X-ray Microanalysis in the Scanning Electron Microscope
}

\author{
Raynald Gauvin ${ }^{1}$, Eric Lifshin ${ }^{2}$, Hendrix Demers ${ }^{1}$, Paula Horny ${ }^{1}$ and Helen Campbell ${ }^{1}$ \\ 1: Department Metals and Materials Engineering, McGill University, Montréal, Québec, \\ Canada, H3A 2B2. \\ 2: Albany Institute for Materials, CESTM, 251 Fuller Road, Albany, NY, 12301, USA.
}

The computation of characteristic lines as well as the bremstrahlung allows to improve the accuracy of x-ray microanalysis in the scanning electron microscope (SEM) using an energy dispersive spectrometer (EDS). This entitles not only to model background's shape for the extraction of net X-ray intensity, but also to predict peak to background ratios and delectability limits of an element in a given system. Also, the magnitude of fluorescence correction from the absorption of characteristic lines and the bremstrallung can be computed from simulated X-ray spectra. Finally, new ways to perform quantitative analysis could be developed since the total background intensity is function of specimen's composition.

In this paper, a new Monte Carlo program that computes the full X-ray spectrum, measured with an EDS detector in a SEM is described. This program, named Win X-ray, is based on the simulation of electron scattering in solids using the Monte Carlo method. This technique is described by Hovington et al. ${ }^{1}$ for X-ray microanalysis in the SEM. The complete details of this program are given in the paper of Gauvin et al. ${ }^{2}$. Win X-ray have been designed to simulate the full X-ray spectra (the characteristic lines and the bremstrahlung) for homogeneous alloys or compounds for any angles of the incident electron beam and the X-ray detector axis relative to the specimen normal. Also, this program computes absolute X-ray intensities in order to simulate real experimental conditions for incident electron energies ranging from 1 to $40 \mathrm{keV}$. This program also computes the complete X-ray spectrum of a non conductive material using the model described by Hendrix et al. ${ }^{3}$.The Monte Carlo program Win X-ray can be downloaded for free at www.minmet.mcgill.ca/MonteCarlo. Figure [1] shows the window interface of Win X-ray.

\section{References}

1. P. Hovington, D. Drouin and R. Gauvin (1997), "Casino: A New Era of Monte Carlo Code in C Language for Electron Beam Interaction, Part I: Description of the Program", Scanning, Vol.19, pp. 1-14.

2. R. Gauvin, E. Lifshin, H. Demers, P. Horny and H. Campbell (2003), "Win X-ray, a new Monte Carlo Program that Computes X-ray Spectrum for X-ray Microanalysis in the Scanning Electron Microscope", Submitted to Microscopy \& Microanalysis.

3. H. Demers and R. Gauvin (2002), "X-Ray Microanalysis of a Coated NonConductive Specimen: Monte Carlo Simulations", Microscopy \& Microanalysis, Vol. 8, Supp. 2, pp. $1462-1463$. 


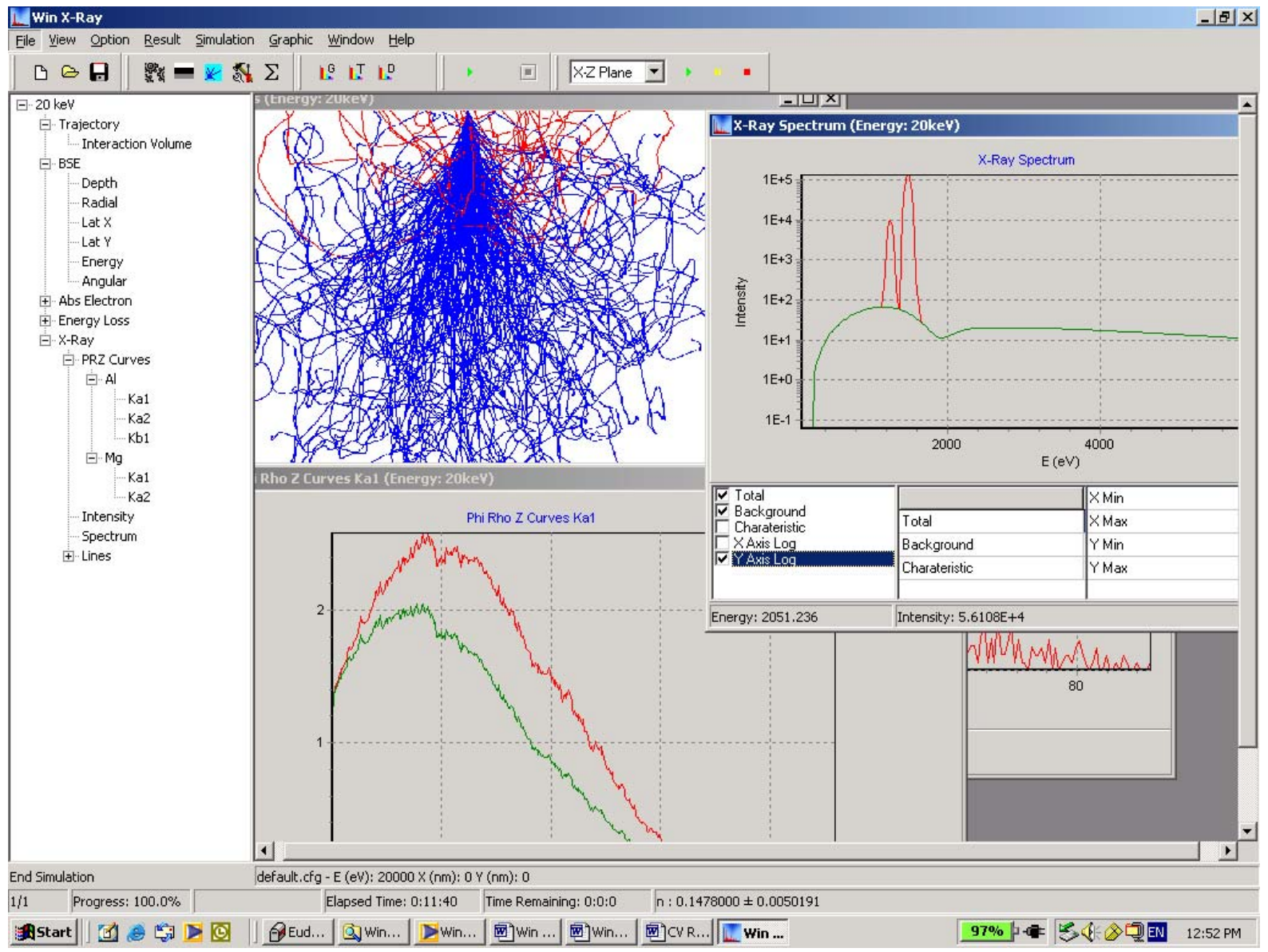

Figure [1] Window interface of the Win X-ray Monte Carlo program. 